\title{
Vivências do familiar frente ao diagnóstico de diabetes mellitus na criança/adolescente
}

\author{
Family experiences in the diagnosis of diabetes mellitus in children/adolescents \\ Vivencias del familiar frente al diagnóstico de diabetes mellitus en el \\ niño/adolescente
}

Gomes, Giovana Calcagno루 ${ }^{1}$ Moreira, Moara Avila de Jesus²; Silva, Camila Daiane³; Mota, Marina Soares ${ }^{4}$; Nobre, Camila Magroski Goulart5; Rodrigues, Eloisa da Fonseca ${ }^{6}$

Como citar este artigo: Gomes GC, Moreira MAJ, Silva CD, Mota MS, Nobre, CMG, Rodrigues EF. Vivências do familiar frente ao diagnóstico de diabetes mellitus na criança/adolescente. J. nurs. health. 2019;9(1):e199108

\section{RESUMO}

Objetivo: identificar as vivências do familiar frente ao diagnóstico de Diabetes Mellitus na criança/adolescente. Métodos: pesquisa qualitativa, realizada com 15 familiares de crianças/adolescentes do Centro Integrado de Diabetes de um Hospital Universitário do sul do Brasil. Coletou-se os dados no primeiro semestre de 2014 por entrevistas semiestruturadas, tratadas pela análise de Conteúdo. Resultados: os familiares identificam os sinais/sintomas, mas não associados com diabetes ou confundidos com outras patologias. 0 diagnóstico ocorre em consultas de rotina ou quando ocorre o agravamento da situação. Os sentimentos comuns são culpa e medo, por não saber como agir. Primeiro ocorre a negação e depois a busca por conhecimento acerca da doença. Considerações Finais: ao conhecer esse processo, os profissionais de enfermagem podem contribuir para diminuir o impacto do diagnóstico, apoiando a família e criança/adolescente no enfrentamento de mudanças.

Descritores: Diabetes mellitus; Família; Criança; Adolescente; Enfermagem.

\section{ABSTRACT}

Objective: to identify the experiences of the relative in the diagnosis of Diabetes Mellitus in the child/adolescent. Methods: qualitative research, carried out with 15 family members of children/adolescents from the Integrated Diabetes Center of a University Hospital in southern Brazil. Data was collected in the first half of 2014 through semi-structured interviews, handled by Content analysis. Results: family members identify the signs/symptoms, but they do not associate it with

1 Enfermeira. Doutora em Enfermagem. Universidade Federal do Rio Grande (FURG). E-mail: giovanacalcagno@furg.br http://orcid.org/0000-0002-2464-1537

2 Enfermeira. Mestre em Enfermagem. Hospital do Coração do Brasil, Brasília-DF. E-mail: moarajesus@yahoo.com.br http://orcid.org/0000-0001-8286-4440

3 Enfermeira. Doutora em Enfermagem. Universidade Federal do Rio Grande (FURG). E-mail: camilad.silva@yahoo.com.br http://orcid.org/0000-0002-0739-4984

4 Enfermeira. Doutora em Enfermagem. Universidade Federal do Rio Grande (FURG). E-mail: marinamota@furg.br http://orcid.org/0000-0002-5717-9406

5 Enfermeira. Mestre em enfermagem. Prefeitura Municipal do Rio Grande, Rio Grande-RS. E-mail: kamy_magroski@yahoo.com.br http://orcid.org/0000-0002-0163-1352

6 Enfermeira. Mestre em Enfermagem. Universidade Federal do Rio Grande (FURG). E-mail: eloisadafonsecarodrigues@yahoo.com.br http://orcid.org/0000-0002-5052-4086 


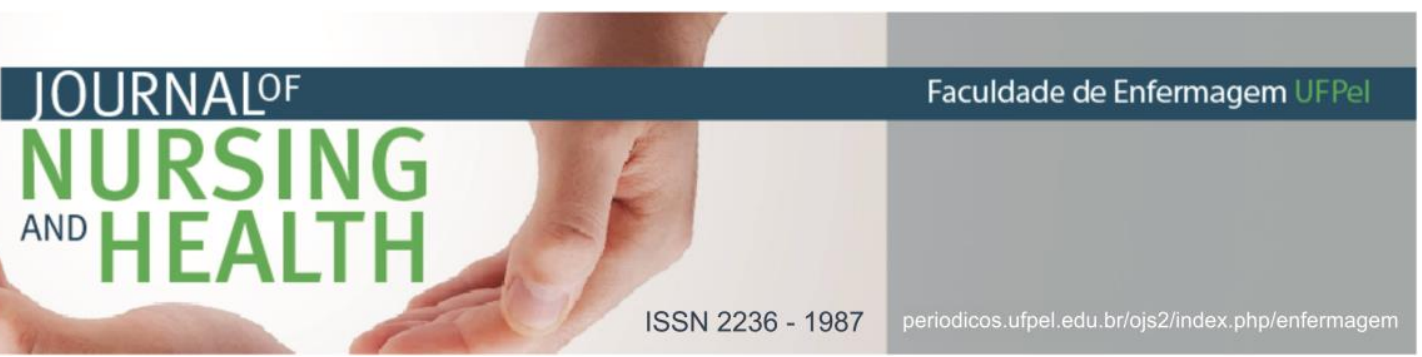

diabetes, or they are confused with other pathologies. The diagnosis occurs in routine consultations or when the situation worsens. Common feelings are guilt and fear, for not knowing how to act. First there is denial and then the search for knowledge about the disease. Final considerations: by knowing this process, nursing professionals can contribute to decrease the impact of diagnosis, supporting the family and child/adolescent in coping with changes.

Descriptors: Diabetes mellitus; Family; Child; Adolescent; Nursing.

\section{RESUMEN}

Objetivo: identificar las vivencias del familiar frente al diagnóstico de Diabetes Mellitus en el niño/adolescente. Métodos: investigación cualitativa, realizada con 15 familiares de niños/adolescentes del Centro Integrado de Diabetes de un Hospital Universitario del sur de Brasil. Se recogieron los datos en el primer semestre de 2014 por entrevistas semiestructuradas, tratadas por el análisis de Contenido. Resultados: los familiares identifican los signos/síntomas, pero no lo asocian con la diabetes o se confunden con otras patologías. El diagnóstico ocurre en consultas de rutina o cuando ocurre el agravamiento de la situación. Los sentimientos comunes son culpa y miedo, por no saber cómo actuar. Primero ocurre la negación y luego la búsqueda por conocimiento acerca de la enfermedad. Consideraciones finales: al conocer este proceso, los profesionales de enfermería pueden contribuir a disminuir el impacto del diagnóstico, apoyando a la familia y niño/adolescente en el enfrentamiento de cambios.

Descriptores: Diabetes mellitus; Familia; Ninõ; Adolescente; Enfermería.

\section{INTRODUÇÃO}

Cerca de 347 milhões de pessoas mundialmente possuem Diabetes Mellitus (DM), no entanto, metade delas desconhecem esse fato, ou seja, não foram diagnosticadas. ${ }^{1-2}$ Cada quatro de cinco pessoas com DM vive em países de baixa/média renda e metade das pessoas que morrem de DM possuem 60 anos ou mais. Estima-se que 4,8 milhões de pessoas morreram em 2012 em decorrência do DM e 471 milhões de dólares foram gastos com o tratamento de portadores. Estima-se que o DM será a sétima principal causa de morte em 2030. ${ }^{2}$ Estes números são gritantes e deixam o alerta para esta doença crônica não transmissível.

No ano de 2013 o número estimado de diabéticos no Brasil foi de 11.934 milhões de pessoas, estando em quarto lugar geral no mundo. Cerca de 10,4 mil crianças e adolescentes entre zero e 14 anos foram diagnosticados com DM tipo 1 no Brasil. ${ }^{3}$
A vivência de uma doença crônica, como o DM, na infância e adolescência, torna-se motivo de diversos conflitos. O cotidiano do portador da doença é modificado com diversas medicações, verificações regulares de glicemia, hospitalizações para exames e tratamento, além de enfrentar limitações, principalmente físicas e alimentares. ${ }^{4}$

A criança/adolescente com DM também possui seu cotidiano afetado, necessitando de um olhar e um cuidado diferenciado. Uma pesquisa realizada com adolescentes, verificou que a exatidão para ingestão dos medicamentos e as cobranças dos pais e profissionais de saúde figuram entre as dificuldades vivenciadas com o DM. ${ }^{5}$

Nesse cenário a família exerce papel fundamental para a manutenção do equilíbrio emocional da criança/adolescente. A maneira como a família trata a situação e o 
conhecimento que possui sobre a patologia, influenciará a criança/adolescente a aceitar ou negar, bem como a compreender as limitações e não se revoltar com a doença. ${ }^{6}$ Além da família, os profissionais também possuem participação nas vivências da criança/adolescente frente ao diagnóstico de DM. A enfermagem, especialmente, deverá prestar uma assistência integral diferenciada envolvendo ações de educação em saúde, para melhor adesão ao tratamento necessário.

Nesse sentido, compreender como o familiar vivencia o processo de diagnóstico de DM pode contribuir para a atuação profissional do enfermeiro, dando o suporte necessário para a melhor aceitação e adesão da criança/adolescente ao tratamento. Desta forma, justifica-se o estudo na possibilidade de melhorar as práticas de cuidado de enfermeiros direcionadas a esse grupo, bem como na construção do conhecimento sob a ótica do familiar.

Assim, questionou-se: Quais as vivências do familiar frente ao diagnóstico de Diabetes Mellitus na criança/adolescente? Desta forma, objetivou-se identificar as vivências do familiar frente ao diagnóstico de Diabetes Mellitus na criança/adolescente.

\section{MATERIAIS E MÉTODOS}

Realizou-se uma pesquisa descritiva, exploratória de cunho qualitativo, no Centro Integrado de Diabetes (CID) de um Hospital Universitário do sul do país. Justifica a escolha deste cenário por se tratar de um centro de referência, de extrema relevância para a população local, no qual poucas pesquisas haviam sido realizadas.

Participaram 15 familiares de crianças/adolescentes com DM que atenderam aos critérios de inclusão: ser o principal familiar cuidador, ter mais que 18 anos, ter a criança/adolescente sido atendido no CID, acompanhar a criança/adolescente durante suas consultas, prestar-lhe cuidados diretos, ou seja, estar diariamente envolvido no cuidado domiciliar seja na verificação da glicemia, alimentação ou uso de medicações, entre outros. Foram excluídos do estudo os familiares cuidadores principais que, no momento da coleta dos dados, não estavam acompanhando a criança/adolescente na consulta. Destaca-se que nenhum pai esteve presente, acompanhando a criança/adolescente.

A coleta de dados ocorreu no primeiro semestre de 2014, por meio de entrevistas semiestruturadas únicas com cada participante, obtendo-se uma melhor compreensão da realidade relativa ao fenômeno em estudo. Foi operacionalizada por meio de um roteiro com perguntas acerca das pessoas/serviços de saúde, do cuidado familiar às crianças/adolescentes com Diabetes Mellitus, do momento de recebimento do diagnóstico. As entrevistas foram realizadas em um consultório no CID, pois garantia conforto e privacidade, utilizou-se um gravador e a duração média foi de 30 minutos.

Para o tratamento dos dados, utilizou-se a técnica de Análise de 


\section{JOURNALOF \\ NURSING \\ ${ }^{\text {AND HEALTH }}$}

ISSN 2236 - 1987

do DM, como poliúria, polifagia, emagrecimento, apatia, sonolência.

Para nós ele ficou doente, emagreceu, teve todos aqueles sintomas, perdeu peso. Ficou muito desanimado. Urinava muito, bebia muita água. Isso em questão de uma semana. (F9, 3a)

Começou a dor na barriga, ter vômitos, urinar na cama, a ter muito sono [...]. Deram morfina para ele. Porque ele tinha muita dor na barriga e vômitos. Começou a ter muita sede também, mas achávamos que era infecção intestinal. (F5, 14a)

Ele não tomava água, só que ele começou a tomar [água] demais, demais. Teve um dia que eu trabalhei a noite, quando eu cheguei minha mãe disse que ele tomou água a noite inteira, não é normal isso, água e guaraná. Ele tinha na época nove anos. Eu pensei, que estranho. (F1, 11a)

Apesar das mudanças percebidas pelo familiar na criança/adolescente, não desconfiavam da possibilidade de ser uma doença crônica e nem imaginavam a importância dos cuidados necessários. A influência de pessoas próximas dificulta a percepção sobre a saúde da criança/adolescente.

A minha sogra dizia: - Não te preocupa, criança que come bem não tem doença. A minha mãe também [dizia]. Eu fui atrás daquilo que elas diziam e fui deixando. (F8, 16a) 


\section{ISSN $2236-1987$}

Mesmo sendo profissional da área da saúde, mas como mãe [...] não enxerga. Na verdade, estava na minha cara, estava estampado, [...]. Todo tempo ele urinava, tomava água. Então, estava na minha cara. Foi um pavor, um pavor. (F1, 11a)

Com a continuidade dos sintomas, - familiar passou a desconfiar que a criança/adolescente poderia estar apresentando algum problema de saúde grave.

[...] Dois dias depois ele estava pior, muito desanimado, só deitado achamos que era anemia e dávamos comida para ele. De forma alguma se imaginava. Pensávamos que ele tinha alguma anemia, porque ele emagreceu muito e pelo desânimo. Não tínhamos nenhum caso assim próximo, de Diabetes. (F9, 3a)

É frente a permanência e agravamento dos sintomas que 0 familiar busca o serviço de saúde. Esse é o momento em que o familiar se depara com a notícia que confirma todos os sintomas percebidos na criança/adolescente.

Quando eu cheguei no médico, a primeira coisa que fizeram foi um HGT [hemoglicoteste], e estava com 469 [mg/dl]. Ele internou na mesma hora. Foram feitos os exames e foi diagnosticado Diabetes Mellitus tipo 1. Foi um susto muito grande. [...] e ainda a minha mãe leiga dizia para mim: - Ele tinha que fazer aquele exame do dedo. E eu dizia para ela que era loucura, dei risada da cara dela! Que loucura! Imagina o meu filho ter Diabetes! (F1, 11a)

\section{O diagnóstico de Diabetes} Mellitus na criança/adolescente

Nem sempre levar a criança/adolescente ao serviço de saúde significa receber o diagnóstico de imediato. Alguns familiares vivenciaram a dificuldade de receber a confirmação do diagnóstico de DM.

A princípio ele estava com uma infecção na garganta, consultou e estava tomando antibiótico, mas foi piorando [...] levamos ao hospital e lá os médicos disseram que era apendicite, que iriam operar. A outra médica que estava de plantão, resolveu dizer que ele poderia estar com um tumor cerebral. Foram fazer uma ressonância e descartaram. [...] mas ele parou de caminhar... Diziam que ele estava delirando devido a morfina. Me trataram muito mal. [...] lá pelas tantas eu pedi o teste da glicose. A enfermeira fez, e então viram que não era apendicite e sim diabetes, deram insulina para ele. ( $\mathrm{F} 5,14 \mathrm{a})$

No início os médicos não diagnosticaram. Ficaram uns dez dias com essa história de que poderia ser uma alergia, deram remédio. Ele estava sentadinho no sofá e passou mal. Caiu e eu achei que era um desmaio, porque na minha família não tem ninguém [com diabetes]. Eu não tinha noção, nem conhecimento desse problema de Diabetes. (F14, 14a) 


\section{JOURNALOF \\ NURSING \\ ${ }^{\text {AND HEALTH }}$}

Outros familiares receberam a notícia do diagnóstico de DM em uma consulta de rotina ou por outro motivo que não os sintomas de DM.

Nós o levamos para rotina no posto e elas fizeram o teste nele. A enfermeira repetiu porque não acreditou. Disse que estava marcando mais de 600. Chamou a médica e ali ela me deu o diagnóstico e encaminhou ele direto para o hospital. (F4, 7a)

Ele teve uma infecção urinária que não curava. Fomos no pediatra que mandou ele para casa. Ficava vermelho na região do pênis. Ele teve febre e urinava muito. O médico desconfiou, fez o HGT e deu alto. (F3, 11a)

Independente de quando o familiar recebeu o diagnóstico de DM, o impacto foi grande, causando diversos sentimentos e reações. 0 fato de a criança/adolescente estar em crise e necessitar de hospitalização, agrava o recebimento do diagnóstico. Em alguns casos o familiar sente culpa pelo adoecimento da criança/adolescente.

O médico disse: - Pois é, seu filho é diabético, a glicose dele está 585 [mg/dl], vamos ter que levar para o hospital [...]. Na hora eu fui forte [...]. Eu liguei para a minha família para dizer que eu estava indo para o hospital, aquela coisa toda de ambulância. Eu desabei porque era grave. Chorei e tudo. (F9, 3a)

Quem recebeu o primeiro susto fui eu, porque eu que estava com ele. Já saiu dali direto para o hospital para internação. Eu me desesperei muito, muito mesmo. Eu chorava muito, eu não aceitava [...]. (F4, 7a)

Saiu direto para a UTI do hospital. Ele estava muito mal, não parava em pé, não abria os olhos. [...] foi um choque. A nossa vida parou naquele momento. Eu sabia que essa doença é muito grave. Ele foi diagnosticado durante a crise. (F7, 16a)

No primeiro momento eu nem pensei em nada, eu só chorei [...]. Logo que eu soube o diagnóstico, eu fiquei sem chão. Me deu um sentimento de culpa. Achei que eu fosse a culpada por ter chegado a esse ponto. (F15, 2a)

Para alguns familiares, 0 recebimento do diagnóstico de DM na criança/adolescente é agravado pela ocorrência de outros acontecimentos no espaço privado, tornando a situação mais traumática. Destacam-se situações como hospitalizações, cirurgias, gestação e morte na família.

Teve um ano em que ela teve cinco internações. Foi muito difícil. Junto com o Diabetes veio a epilepsia. (F6, 14a)

Naquele ano, tínhamos perdido duas pessoas da família em agosto, 15 dias de diferença, e em dezembro o meu filho se hospitaliza. Todo mundo se apavorou. A família, teve uma 


\section{ISSN $2236-1987$}

reação pior do que a nossa. Não aceitam até hoje. ( $\mathrm{F9}, 3 \mathrm{a})$

Ele teve que fazer uma cirurgia nos olhos, porque ele tinha catarata congênita, pois nasceu prematuro. Só que com o aumento da glicose, ele começou a não enxergar nada, ficar turva $a$ visão. $O$ endócrino disse para cuidarmos o fundo de olho, os pés, tudo dele. $(\mathrm{F} 13,11 \mathrm{a})$

A preocupação com a criança/adolescente pode ser tão grande, que afeta toda a família. 0 medo de não saber lidar com a situação ou ter os cuidados necessários é constante. Tal sentimento pode estar associado ao desconhecimento sobre a patologia.

Eu tinha que segurar a barra dele [criança/adolescente], da minha mãe, do meu pai e mais a minha sogra. (F1, 11a)

Chorávamos muito, tanto eu como ele [marido], porque achamos que iriamos perder ele. Não conhecíamos nada sobre diabetes. $(\mathrm{F} 12,6 \mathrm{a})$

O meu marido teve medo de não conseguir fazer as coisas nele, os testes, as insulinas, mas agora ele já está fazendo. (F15, 2a)

\section{O familiar e o conhecimento sobre Diabetes Mellitus}

Mesmo ciente do diagnóstico de DM da criança/adolescente, o familiar pode usar de mecanismos de defesa, como a negação e o desejo de não aprender sobre a doença. Muitos familiares tinham esperança de que tudo era momentâneo.

As enfermeiras lá me explicavam, me ensinavam como se aplicava a Insulina. E eu dizia: - Não, eu não quero aprender. Eu tinha uma força, mas ao mesmo tempo eu achava que aquilo ali iria passar, que era impossível que aquilo seria para sempre. Porque eu nunca tinha escutado falar de Diabetes tipo 1. Eu não sabia que aquilo ali era para sempre. Quando eles falaram que meu filho estava com Diabetes, eu pensei: - É um momento. Vai passar. (F14, 14a)

Por outro lado, frente ao diagnóstico de DM, o familiar tenta buscar por conhecimento sobre a patologia em diversas fontes, como a internet ou casos com pessoas próximas. No entanto, adquirir o conhecimento desta maneira pode agravar o sentimento do familiar, em relação as expectativas de vida e evolução da doença.

Ficamos muito assustados porque depois que começamos a pesquisar e prestar atenção na televisão, víamos as pessoas amputadas, com infarto, cegas, com problemas de rins. Começamos a pensar: -Minha filha vai ficar de cadeira de rodas, ela pode ficar cega, ela vai ficar sem rim. Chegou ao ponto de não entrar mais açúcar em casa. (F6, 14a)

Veio um monte de coisas na cabeça porque por parte da minha mãe, eu perdi meu avô por 
causa do Diabetes. Então, vem aquilo, vem a cegueira, vem tudo. (F4, 7a)

Alguns familiares buscaram 0 conhecimento com os profissionais da saúde, adquirindo informações que subsidiaram $\mathrm{o}$ cuidado da criança/adolescente.

Eu achava até que Diabetes não dava em crianças, eu não sabia nada. E eu achava que Diabetes era uma doença só de adultos [emocionada]. Ficamos quinze dias hospitalizados, ele fazendo a insulina e HGT várias vezes por dia. Eu fui aprendendo os cuidados com as enfermeiras, com os médicos, no hospital mesmo, me ensinaram a fazer. Fui aprendendo assim. (F8, 16a)

Eu lembro da enfermeira da Pediatria. Ela sempre me explicava. Quem estava de plantão no dia era um doutor que eu não esqueço dele nunca. Me explicou exatamente o que era o Diabetes, que eu não poderia sair do hospital sem saber aplicar insulina. Eu não fazia a menor ideia. $(\mathrm{F} 6,14 \mathrm{a})$

\section{DISCUSSÃO}

O familiar é quem primeiro percebe que a criança/adolescente não está bem. Diante dos sintomas apresentados, verifica-se que algo diferente está acontecendo, porém não se sabe explicar ou definir do que se trata. Alguns sintomas característicos do DM como a poliúria, polifagia, emagrecimento e apatia, foram notados pelo familiar na criança/adolescente. Outros sinais e sintomas que o familiar também percebeu foi a mudança de humor, temperamento e sonolência. ${ }^{7}$

Verificou-se que as pessoas próximas ao familiar da criança/adolescente podem interfere na percepção da gravidade dos sintomas de DM. Por outro lado, uma pesquisa realizada em Maringá-PR, com onze pais de crianças e adolescentes com diabetes mellitus tipo 1, identificou uma maior aceitação da doença por parte do familiar quando as pessoas próximas, como parentes e amigos, eram portadoras de DM. ${ }^{8}$

Em alguns casos, o familiar imaginava se tratar de outras doenças que não $0 \mathrm{DM}$, como anemia. Evidenciando, possivelmente uma forma de negação da situação. No entanto, ao agravar os sinais de alerta para DM, o familiar buscou ajuda com profissionais e serviços de saúde. Contudo, nem sempre esse auxílio foi imediato, pois em alguns casos, 0 diagnóstico demorou a ser confirmado.

Dessa forma, o DM pode demorar a ser diagnosticado, contribuindo para o aumento da morbidade da doença e piora da qualidade de vida de seus portadores. A falta de urgência pela equipe de saúde na busca pelo diagnóstico do DM na criança/adolescente é motivo de frustração dos familiares. ${ }^{9}$

A investigação de outras patologias que não a $\mathrm{DM}$, pode levar a diagnósticos precipitados, deixando a família mais apreensiva. Alguns familiares relataram o diagnóstico de tumor, apendicite, entre outros. Erros 


\section{JOURNALOF \\ NURSING \\ AND HEALTH}

ISSN 2236 - 1987

no diagnóstico tardam o início do tratamento e aumentam a aflição dos familiares. Os sentimentos dos pais em relação à doença, como negação e medo, podem ser superados de acordo com os esclarecimentos recebidos. ${ }^{8}$

0 impacto de receber 0 diagnóstico que confirma os sintomas que o familiar vinha percebendo, pode gerar sentimentos negativos como 0 medo e a culpa. O DM por ser uma patologia incurável e por necessitar, em alguns casos, do uso de insulina terapia, pode gerar diferentes sentimentos, principalmente o medo e insegurança. ${ }^{10} \mathrm{Uma}$ pesquisa realizada com pais de crianças com DM, identificou que 0 momento do diagnóstico foi permeado por tristeza, angústia, desespero e sofrimento, bem como a ansiedade de não saber lidar com a nova situação. ${ }^{8}$

Esses sentimentos do familiar podem contribuir para que a criança/adolescente se sinta pouco responsável pela sua saúde. Destaca-se que o empenho e perseverança da família pode contribuir fortemente para ações voltadas à doença. ${ }^{7}$ Ainda, o conhecimento que o familiar possui sobre a doença e a forma como a enfrenta, pode influenciar na aceitação e relacionamento com a criança/adolescente. ${ }^{8}$

Ao se deparar com uma doença crônica as mães entram em desespero e o sentimento mais comum na descoberta do diagnóstico de DM da criança/adolescente é o de negação. ${ }^{10}$ A negação também pode se manifestar na criança/adolescente, principalmente pelas mudanças que ocorrem no cotidiano de vida e pelos prazeres permitidos somente para outras crianças/adolescentes. ${ }^{11}$

Uma pesquisa identificou nos seus resultados que os familiares, mesmo que já tenham recebido o diagnóstico a mais de três anos, possuíam dificuldades no cotidiano com as crianças/adolescente, especialmente no que se referia os hábitos alimentares. ${ }^{11}$ Outro estudo sugeriu que a habilidade da mãe em ser atenciosa e afetiva com a criança/adolescente, pode influenciar no controle glicêmico ruim. ${ }^{12}$

Uma pesquisa realizada com crianças evidenciou a importância de avaliar o conhecimento prévio da criança e da família, buscando esclarecer dúvidas e desmistificando os preconceitos. Aponta ainda, a utilização de estratégias criativas para que a criança exponha seus sentimentos e vivências com a doença. ${ }^{13}$

Além da criança, também é importante conhecer como o adolescente com diabetes interpreta sua condição de saúde e as exigências terapêuticas, contribuindo para a vivência desse período de vida. Uma pesquisa identificou que a maioria dos adolescentes apresentaram um alto nível de reponsabilidades quanto ao autocuidado. ${ }^{14}$ Esta postura demonstra uma melhor aceitação pessoal e a adesão à terapia, podendo contribuir para melhor convívio social.

Alguns familiares receberam 0 diagnóstico de DM em consultas de rotinas ou por outro motivo que não pelos sintomas de DM, outras receberam em um momento de crise com internação. Ainda, o momento do 


\section{JOURNALOF \\ NURSING \\ ${ }^{\text {ANO HEALTH }}$}

ISSN 2236 - 1987

diagnóstico foi agravado quando 0 familiar vinha passando por outras situações difíceis, como mortes, cirurgias, entre outros. Independente do momento, o círculo de amigos, a religião, escola, profissionais de saúde e a mídia social são de extrema importância no apoio e auxílio para a criança e família. ${ }^{15}$

Destaca-se que o familiar sente a necessidade de conhecer melhor a patologia para prestar um melhor cuidado à criança/adolescente. Os familiares afirmaram que o pai tinha medo de administrar insulina na criança/adolescente. Essa realidade também foi identificada em um estudo no qual os pais afirmaram que a aplicação de insulina é o momento mais difícil do tratamento de DM. Esse momento provoca sentimentos de aflição, insegurança, medo e sofrimento nos pais. ${ }^{8}$

Apesar dessas reações e sentimentos gerados pelo diagnóstico de DM, bem como as mudanças necessárias do tratamento, é importante a criança/adolescente aderir ao autocuidado. Assim, a família precisar delegar o controle sobre a saúde, incentivando praticar a monitorização da glicemia e administração de insulina. ${ }^{16} \mathrm{~A}$ família e os profissionais de saúde podem contribuir para que a criança/adolescente desenvolva o autocuidado. A educação em DM deve ser constante e é indispensável para o autoconhecimento e o autocuidado que irá garantir uma melhora na qualidade de vida.

Diante da realidade de receber o diagnóstico de DM, são necessários a união e o apoio de todos que estão próximos, para minimizar o fator estressante presente já que este diagnóstico, geralmente, não é esperado. A equipe de saúde deve aproveitar esse momento para criar a boa relação, laços de confiança e respeito entre família, profissionais e a criança. ${ }^{11}$

Os familiares, principalmente a mãe, buscam por informações e conhecimento sobre a patologia, seja pela internet ou por casos ocorridos com pessoas próximas. Essas, também precisam compreender a doença e dar o suporte necessário para a mãe. Uma pesquisa identificou que os pais esperam uma corresponsabilização dos demais familiares em relação ao controle alimentar da criança. ${ }^{11}$

O familiar relata buscar apoio e conhecimento nos serviços e profissionais de saúde. Neste estudo, enfermeiros e médicos deram o suporte que a família necessitava. No entanto, por vezes, os profissionais e serviços focam sua atenção apenas no controle do DM, deixando de lado as orientações dos cuidados diários com a criança/adolescente. ${ }^{17} \mathrm{~A}$ falta de apoio profissional para a família leva à sensação de desamparo e o enfermeiro pode diminuir o distanciamento construindo uma prática que contribua no enfrentamento das dificuldades. ${ }^{8}$

É preciso que os serviços de saúde, principalmente o enfermeiro, incentive a família a desenvolver o cuidado da criança/adolescente com DM, capacitando-os em relação às limitações e mudanças que a doença exige. ${ }^{11}$ Verifica-se a necessidade de os profissionais de saúde buscarem nas suas práticas de cuidado, incentivar e 


\section{JOURNALOF \\ NURSING \\ AND HEALTH}

ISSN 2236 - 1987

ensinar a família, para que o convívio com DM seja melhor aceito. ${ }^{6}$

\section{CONSIDERAÇÕES FINAIS}

A partir dos resultados foi possível verificar o alcance do objetivo de identificar as vivências do familiar frente ao diagnóstico de Diabetes Mellitus na criança/adolescente. 0 familiar é a primeira a perceber os sinais e sintomas de DM na criança/adolescente, no entanto não os associam à doença ou acreditam ser outra patologia que não o DM. A busca pelos profissionais e serviços de saúde ocorre com o agravamento da situação de saúde/doença

da criança/adolescente. 0 processo de descobrir o diagnóstico de DM, envolveu também algumas dificuldades como a investigação de outras patologias que acarretou na demora da confirmação.

Os sentimentos que envolvem o momento do diagnóstico são negativos, como a culpa e o medo, principalmente por não saberem como lidar com a nova situação da criança/adolescente e com as mudanças geradas pela patologia. Ainda, o familiar passa por um período de negação e de não querer aprender sobre os cuidados exigidos pelo DM. No entanto quando aceitam a situação buscam conhecimento na internet, pessoas próximas e profissionais de saúde.

Enfatiza-se as limitações do estudo, o qual se restringiu a uma única realidade sociocultural, bem como ao gênero feminino, pois o pai não esteve presente durante a realização da coleta de dados. Ainda como limitação, destaca-se que o estudo em tela teve como participantes apenas os familiares envolvidos nos cuidados das crianças/adolescentes com DM, evidenciando a necessidade da realização de futuras pesquisas em que a criança/adolescente sejam o foco de estudo. Torna-se importante que futuras pesquisas priorizem também a participação do pai, para que seus sentimentos e experiência do recebimento do diagnóstico de DM possam ser compartilhados com outros pais na mesma situação.

Destaca-se que conhecer como ocorre 0 processo de receber 0 diagnóstico de DM e os sentimentos do familiar, permite uma atuação de enfermagem qualificada e diferenciada para o suporte tanto da família quanto da criança/adolescente. Assim, os profissionais da saúde podem contribuir de diferentes formas no enfrentamento das necessidades e mudanças exigidas, por meio de ações sensibilizadas pelos sentimentos da família, por esclarecimentos, panfletos informativos, grupos de apoio e/ou rodas de conversa já no momento do diagnóstico ou em consultas de enfermagem de rotina para o acompanhamento da adesão ao tratamento. Ao conhecer o processo de diagnóstico de DM e os sentimentos dos familiares, os profissionais de enfermagem podem contribuir de maneira mais sensibilizada para diminuir o impacto do diagnóstico, bem como apoiar o familiar e a criança/adolescente no enfrentamento das mudanças exigidas pela doença. 


\section{REFERÊNCIAS}

1 International Diabetes Federation (IDF). IDF diabetes atlas: eighth edition 2017 [Internet]. 2017[cited 2018 Nov 08]. Available from: http:/ / diabetesatlas.org/IDF_Diabetes _Atlas_8e_interactive_EN/

2 World Health Organization (WHO). Key facts [Internet]. 2018[cited 2018 Nov 08]. Available from: http://www.who.int/en/newsroom/fact-sheets/detail/diabetes

3 Internacional Diabetes Federation (IDF). What is diabetes [Internet]. 2018[cited 2018 Nov 08]. Available from:

https: / /www.idf.org/aboutdiabetes/ what-is-diabetes

4 Fialho FA, Dias IMAV, Nascimento L, Motta PN, Pereira SG. Crianças e adolescentes com diabetes mellitus: cuidados/implicações para a enfermagem. Rev. baiana enferm. [Internet]. 2011[acesso em 2018 nov 08];25(2):145-54. Disponível em: https://portalseer.ufba.br/index.php /enfermagem/article/view/4820/446 7

5 Ferreira LE, Zanatta EA, Brum MLB, Nothaft SC, Motta MGC. Diabetes mellitus sob a ótica do adolescente. Cogitare enferm. [Internet]. 2013[acesso em 2018 dez 30]; 18(1):71-7. Disponível em:

https://www.redalyc.org/pdf/4836/4 83648961010.pdf

6 Bertin RL, Elizio NPS, Moraes RNT, Medeiros CO, Fiori LS, Ulbrich AZ. Percepções do cotidiano alimentar de crianças e adolescentes com diabetes mellitus tipo 1 Rev. Contexto Saúde (Impr.). [Internet]. 2016[acesso em
ISSN 2236 - 1987

2018 nov 08];16(30):100-9. Disponível em:

https://www.revistas.unijui.edu.br/in dex.php/contextoesaude/article/view $/ 5665$

7 Bardin L. Análise de conteúdo. São Paulo: Edições 70; 2011.

8 Pimentel RRS, Targa T, Scardoelli MGC. From diagnosis to the unknown: perceptions of parents of children and adolescents with diabetes mellitus. Rev. enferm. UFPE on line. [Internet]. 2017[cited 2018 Nov 08];11(3):111826. Available from: https://periodicos.ufpe.br/revistas/r evistaenfermagem/article/view/1348 $6 / 16203$

9 KratzerJ. Structural barriers to coping with type 1 diabetes mellitus in ghana: experiences of diabetic youth and their families. Ghana Med J [Internet]. 2012[cited 2018 Nov 08];46(2):39-45. Available from: https: / /www.ncbi.nlm.nih.gov/pmc/a rticles/PMC3645143/pdf/GMJ462S0039.pdf

10 Okido ACC, Almeida A, Vieira MM, Neves ET, Mello DF, Lima RAG. Care demands of children with type 1 Diabetes Mellitus. Esc. Anna Nery Rev. Enferm. [Internet]. 2017[cited 2018 nov 08];21(2):e20170034. Available from:

http://www.scielo.br/pdf/ean/v21n2 /en_1414-8145-ean-21-02e20170034.pdf

11 Malaquias TSM, Marques CDC, Faria ACP, PupulimJSL, MarconSS, Higarashi $\mathrm{IH}$. The division of the care of the child or adolescent with type i diabetes with the family. Cogitare enferm. [Internet]. 2016[cited 2018 Nov 08];21(1):01-07. Available from: 
https://revistas.ufpr.br/cogitare/arti cle/view/42010/27252

12 Kirchner LF, Benitez P, Tatmatsu DIB, Ribeiro GW, Vargas VM, Del Prette ZAP. Habilidades sociais educativas em mães de crianças/adolescentes com diabetes mellitus tipo 1. Rev. psicol. (Fortaleza, Online). [Internet]. 2017[acesso em 2018 nov 08];8(2):155164. Disponível em: http: / / www.periodicos.ufc.br/psicolo giaufc/article/view/11728/30939

13 Queiroz MV, BritoLMMC, Pennafort VPS, Bezerra FSM. Sensitizing children with diabetes to self-care: contributions to educational practice. Esc. Anna Nery Rev. Enferm. [Internet]. 2016[cited 2018 Nov 08];20(2):337-43. Available from: http://www.scielo.br/pdf/ean/v20n2 /en_1414-8145-ean-20-02-0337.pdf

14 Costa M, Gameiro MGH. Autocuidado dos adolescentes com diabetes mellitus tipo 1: responsabilidade no controlo da doença. Referência. [Internet]. 2016[acesso em 2018 nov 08];4(9):9. $19 . \quad$ Disponível em: http://www.scielo.mec.pt/pdf/ref/vs erlVn9/serlVn9a02.pdf

15 Pennafort VPS, Queiroz MVO, Nascimento LC, Guedes MVC. Network and social support in family care of children with diabetes. Rev. bras. enferm. [Internet]. 2016[cited 2018 Nov 08];69(5):912-9. Available from: http: / /www.scielo.br/pdf/reben/v69 n5/en_0034-7167-reben-69-050912.pdf

16 Greco-Soares JP, Dell'Aglio DD. Relações entre qualidade de vida e diabetes mellitus tipo 1 na adolescência. Contextos Clín.
[Internet]. 2016[acesso em 2018 nov 08];9(2):159-67. Disponível em: http://revistas.unisinos.br/index.php /contextosclinicos/article/view/ctc.2 016.92.02/5654

17 Wolkers PCB, Pina JC, Wernet $M$, Furtado MCC, Mello DF. Acesso, vínculo e continuidade na atenção à saúde da criança com diabetes mellitus em ambulatórios especializados. Atlas CIAIQ2016 [Internet]. 2016[acesso em 2018 nov 08];2:921-25. Disponível em: https://proceedings.ciaiq.org/index.p $\mathrm{hp} /$ ciaiq2016/article/view/838/824

Data de submissão: 05/04/2018

Data de aceite: 16/03/2019

Data de publicação: 02/04/2019 